Research Article

\title{
Fourier Series Analysis for Novel Spatiotemporal Pulse Waves: Normal, Taut, and Slippery Pulse Images
}

\author{
Bo Peng $\left(\mathbb{D},{ }^{1}\right.$ Ching-Hsing Luo $\mathbb{D},{ }^{2}$ Nilotpal Sinha $\mathbb{D},{ }^{1}$ Cheng-Chi Tai $\mathbb{D},{ }^{1}$ Xiaohua Xie $\mathbb{D},{ }^{2}$ \\ and Haiqing Xie $\mathbb{1}^{3}$ \\ ${ }^{1}$ Department of Electrical Engineering, National Cheng Kung University, Tainan 70101, Taiwan \\ ${ }^{2}$ School of Data and Computer Science, Sun Yat-Sen University (East Campus), Guangzhou 510006, China \\ ${ }^{3}$ School of Medical Engineering, Foshan University, Foshan 528000, China
}

Correspondence should be addressed to Ching-Hsing Luo; luojinx5@mail.sysu.edu.cn

Received 7 August 2019; Accepted 23 October 2019; Published 27 November 2019

Academic Editor: Giuseppe Caminiti

Copyright $(92019$ Bo Peng et al. This is an open access article distributed under the Creative Commons Attribution License, which permits unrestricted use, distribution, and reproduction in any medium, provided the original work is properly cited.

In this article, a three-dimensional pulse image (3DPI) instead of a one-dimensional temporal pulse wave is studied to elucidate its spatiotemporal characteristics. To check the spatial and temporal properties of 3DPI, adopted is Fourier series, in which a ratio $(r)$ is defined as one amplitude divided by the sum of the first three amplitudes of harmonics. A ratio sequence is constituted from 70 to 90 ratios in a heartbeat with 70-90 3DPIs by sampling. Twenty-four subjects (14 males and 10 females with age of $22.2 \pm 3.7$ years, $20.4 \pm 1.4 \mathrm{BMI}$, and $112.1 \pm 4.7 \mathrm{mmHg}$ systolic blood pressure) are involved in this research. There are significant statistical differences in the groups of the normal, taut, and slippery 3DPIs by the first harmonic ratio average $\left(\overline{r_{1}}\right)$ and ratio difference $\left(\Delta r_{1}\right)$ produced from the ratio sequence. The proposed method of this study gives us a novel viewpoint to clarify the spatiotemporal characteristics of pulse images, which can translate and quantize the pulse feeling in Chinese medicine texts.

\section{Introduction}

Chinese medicine has been developed for over two thousand years. Pulse diagnosis in Chinese medicine provides a noninvasive method based on the radial pulse feeling of the fingers with temporal and spatial characteristics. In order to measure and analyze these pulses, several pulse diagnostic instruments (PDIs) were developed based on array sensors for detecting not only the temporal characteristics but the spatial characteristics of the pulse [1-3]. Luo and colleagues [4-6] used the PDI with tactile array sensors to get the radial artery pressure signal transformed into a visualized threedimensional waveform, called a three-dimensional pulse image (3DPI). Three types of pulse feeling have been well transformed into 3DPI: normal pulse, taut pulse, and slippery pulse [7].

The signal analysis of 3DPI has been gradually paid attention in recent years. Cui et al. [8] proposed a method for distinguishing taut pulse from normal and slippery pulse based on array pulse volume (APV), defined as the average volume of signals in a pulse cycle. Su et al. [9] suggested to conveniently distinguish normal and taut pulses by contour analysis of the spatial structure, and Luo et al. [10] proposed the L-cube polynomial to describe the 3DPI patterns of these two types.

There are a lot of analytic methods in single pulse wave analysis (PWA) [11-24], of which fast Fourier transform (FFT), Hilbert-Huang transform (HHT), wavelet transform (WT), etc. are efficient ways of viewing waveforms in frequency or time domain. However, it is shown that Fourier series can well present the component of pulse waves given the periodicity of the cardiovascular system [25-30]. Since the pulse signal in a short period of time can be treated as a periodic signal due to its small nonlinearity $[27,31]$, Fourier series is considered a unique and powerful pulse analysis method. Given that the spatial properties of the pulse need to be further analyzed, this study intends to determine the features of three pulse types using Fourier series expanding from one-dimensional to three-dimensional pulse analysis. 


\section{Materials and Methods}

2.1. Experimental Data. The pulse data in this research were collected from healthy subjects using the PDI (IRB no: A-BR-100-166). The subjects included 14 males and $10 \mathrm{fe}-$ males from college students (Table 1). The target three types of pulse were first verified by Chinese medicine practitioners' palpation based on the Chinese medicine theory [32], and finally confirmed by the peripheral augmentation index (pAI), which was defined as the ratio of percussion and tidal wave as well as regularly used in Chinese medicine pulse pattern research $[9,21,33-35]$. Normal pulse data were obtained from these subjects in normal state, taut pulse images were induced by cold pressor test (CPT) $[9,36,37]$, while slippery pulse images were measured from woman subjects during the menstrual cycle [7]. The pulse wave signals at the left wrist on a participant were recorded by the tactile array sensor on position "Guan" of the PDI [1]. The PDI thus continuously recorded 12-channel pulse wave signals during the CPT for each participant. There are three robot fingertips in the PDI with 12-point $(3 \times 4)$ sensors on their tips, and each 12-point tactile sensor array has the size of $1 \mathrm{~cm} \times 0.75 \mathrm{~cm}$ with $100 \mathrm{~Hz}$ sampling rate $[1,5,7,38]$.

2.2. Experimental Procedure. A flowchart (Figure 1) is given to show the way to conduct pulse image analysis. First, we took the readings from the sensor arrays in the form of a column matrix with a sampling frequency of $100 \mathrm{~Hz}$. The 0.1-20 Hz bandpass Butterworth filter was used to reduce the noise, and baseline drift was greatly decreased by wavelet transform [38, 39]. 3DPI is formed by using a cubic interpolation algorithm to expand graphic data points [40], and a two-dimensional pulse image (2DPI) is formed from 3DPI by taking the middle pulse data along the center of the radial artery. Fourier series was adopted to analyze the radial pulse images.

Figure 2 shows a multidimensional pulse image: 3DPI (upper panel) at the peak pulse and d2DPI (dynamic 2DPI, lower panel) of one beat for (a) normal, (b) taut, and (c) slippery pulses. The d2DPI clearly demonstrates the temporal response with one spatial information of array pulse signals, while 3DPI just presents two spatial data without temporal responses in the figure.

In Figure 3(a), d2PDI of a slippery wave is further clearly demonstrated by temporal and spatial responses separately, in which the temporal pulse in one beat is same as the regular single pulse wave, and the wave shape of the spatial pulse is presented along the center of the radial artery. Two spatial peaks move up and down alternately in a slippery 3DPI shown in Figure 3 marked with a triangle and a rectangle.

The first peak moves to its maximum $\left(P_{1 \max }\right)$ at length $=1$ in Figure 3(b) (black rectangle) and time $=0.07$ in Figure 3(d) (white circle), while the second peak reaches its maximum $\left(P_{2 \max }\right)$ at length $=0.34$ in Figure $3(\mathrm{c})$ (black triangle) and time $=0.10$ in Figure $3(\mathrm{~d})$ (black circle). It is especially noted that only one peak exists in the temporal wave while two peaks appear in the spatial wave for a slippery 3DPI or d2DPI.
TABLE 1: Basic physiological data of the subjects.

\begin{tabular}{lc}
\hline Characteristic (unit) & Number or mean \pm SD \\
\hline Number & $24($ male $=14$, \\
Age $($ years) & female $=10)$ \\
BMI $\left(\mathrm{kg} / \mathrm{m}^{2}\right)$ & $22.2 \pm 3.7$ \\
Systolic/diastolic blood pressure & $20.4 \pm 1.4$ \\
(mmHg) & $112.1 / 74.0 \pm 4.7 / 3.4$ \\
Heart rate (beats/min) & $76.0 \pm 5.4$ \\
\hline
\end{tabular}

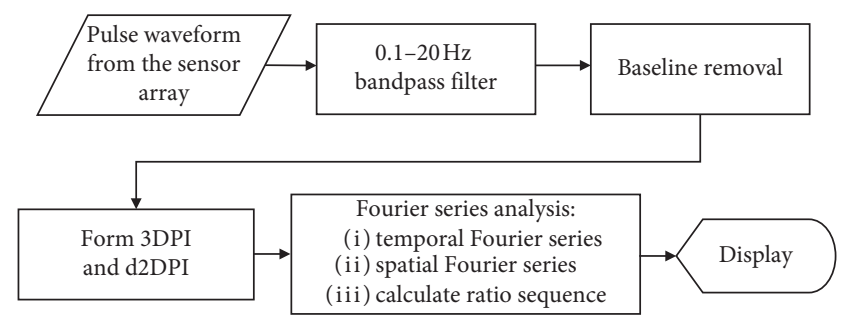

FIGURE 1: Flowchart for pulse image processing.

2.3. Primary Algorithm. A general Fourier series analysis is applied to calculate the temporal or spatial harmonic components of normal, taut, and slippery d2DPIs shown below:

$$
f(x)=\sum_{n=-\infty}^{\infty} C_{n} e^{j(2 \pi n x / X)},
$$

where

$$
C_{n}=\frac{1}{X} \int_{0}^{X} f(x) e^{-j(2 \pi n x / X)} \mathrm{d} x
$$

$C_{n}$ is the amplitude of the temporal or spatial harmonics for $x$ and $X=$ time or length separately.

Fourier series with seven or eight harmonics is empirically adequate for $99.5 \%$ of the variance for one-dimensional temporal wave [41, 42], while the qualified harmonic number of spatial Fourier series is educed to three, on account of the simplicity of the spatial wave shape in this study.

\section{Results and Discussion}

3.1. Temporal Fourier Series. Figure 4 shows the amplitudes of the temporal Fourier series for normal, taut, and slippery pulse images separately. Even there is a little variation in these three temporal pulse waves, but their harmonic amplitudes make no remarkable changes.

3.2. Spatial Fourier Series. Figures 5-6 presents the amplitudes of spatial Fourier series for normal and taut pulse images. By neglecting the base amplitude at $0 \mathrm{~Hz}\left(C_{0}\right)$, the first $\left(C_{1}\right)$ and second $\left(C_{2}\right)$ harmonics show a remarkable difference in amplitudes between normal and taut pulse images. $C_{1}$ is much bigger than $C_{2}$ at normal pulse image, while $C_{1}$ is almost equal to $C_{2}$ at taut pulse image. At taut pulse image, most of the amplitude is accumulated at the 

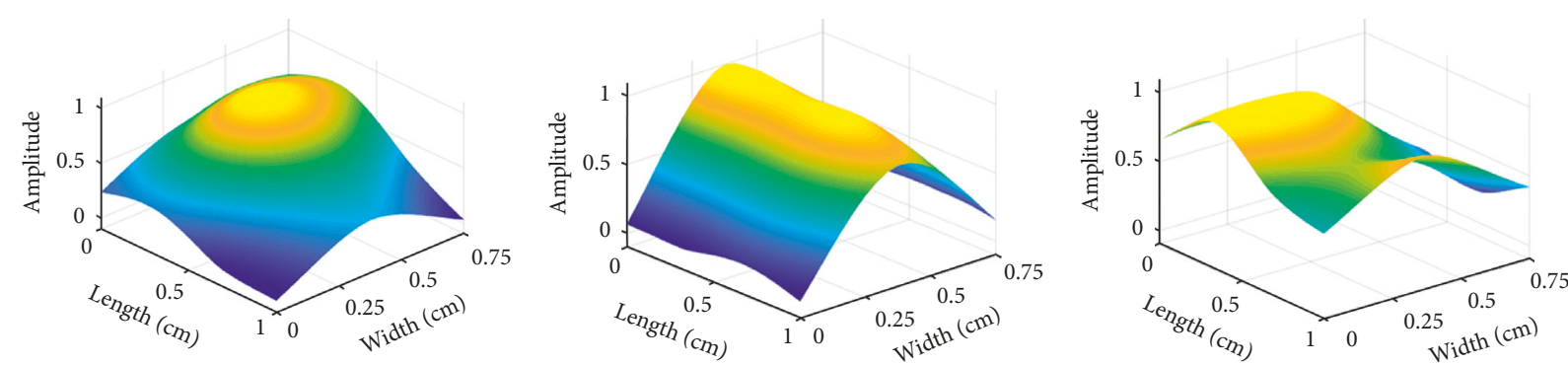

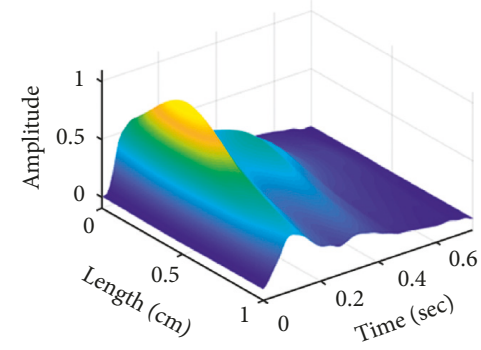

(a)

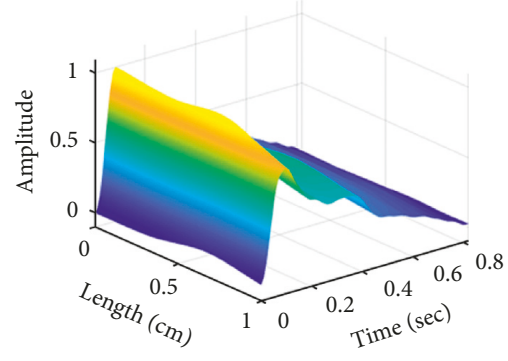

(b)

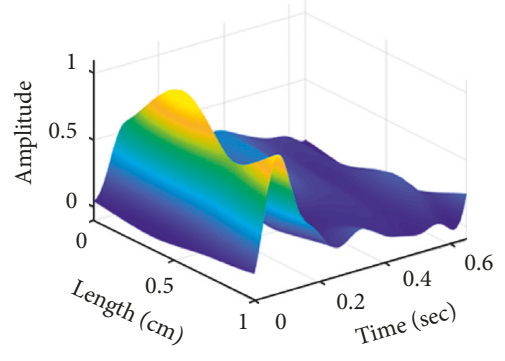

(c)

FIGURE 2: Multidimensional pulse images: (a) normal pulse, (b) taut pulse, (c) and slippery pulse: upper panel, 3DPI; lower panel, d2DPI.

base because the taut pulse image looks like a rectangular pulse with little harmonics in the spatial length along the radial artery.

Figures 7-8 show the spatial Fourier series at the first and second peak maximums $\left(P_{1 \max }\right.$ and $P_{2 \max }$ ) of slippery pulse images separately. For the spatial wave at $P_{1 \max }$, its $C_{1}$ is greater than $C_{2}$ in Figure 7, while for the spatial wave at $P_{2 \max }$, its $C_{1}$ becomes smaller than $C_{2}$ in Figure 8.

3.3. Spatial Harmonic Ratio Sequence. To make the recognition among these three pulse images, harmonic ratios $\left(r_{0}\right.$, $r_{1}$, and $r_{2}$ ) are defined as follows:

$$
\begin{aligned}
& \mathrm{r}_{0}=\frac{C_{0}}{C_{0}+C_{1}+C_{2}}, \\
& \mathrm{r}_{1}=\frac{C_{1}}{C_{0}+C_{1}+C_{2}}, \\
& \mathrm{r}_{2}=\frac{C_{2}}{C_{0}+C_{1}+C_{2}},
\end{aligned}
$$

where $C_{0}, C_{1}$, and $C_{2}$ are the amplitudes of the base, first, and second harmonics in the spatial Fourier series.

For a cardiac cycle, we can get a spatial harmonic ratio sequence from d2DPI. Figure 9 demonstrates three spatial harmonic ratio sequences of consecutive three beats for normal, taut, and slippery pulses. To avoid noise interference at small amplitudes, ratio sequences are not calculated for the pulse with its peak less than 5\% of peak maximum [27]. The harmonic ratio sequence of normal pulses in Figure 9(a) is well distributed without any intersection. For taut pulses in Figure 9(b), the base ratio sequence is always far beyond the first and second harmonic ratio sequences which are almost merged together. Regarding slippery pulses in Figure 9(c), its first and second harmonic ratio sequences intersect each other at the upstroke phase due to two peaks oscillating alternatively. Occasionally, the first harmonic ratio sequence becomes high enough to interact with the base harmonic ratio sequence. Apparently, the behavior of three harmonic ratio sequence showing remarkable differences creates the capability of the recognition among normal, taut, and slippery pulse images (Table 2).

3.4. Statistics. In this study, the first harmonic ratio average $\overline{r_{1}}$ is adopted to represent spatial features of the pulse image, which is the average of $r_{1}$ sequence in one heartbeat. The ratio difference $\Delta r_{1}$ is provided by the difference between $\overline{r_{1}}$ and the $r_{1}$ at the peak of the pulse $\left(r_{1 \mathrm{p}}\right)$, which is more obvious in slippery cases than others (Figure 10). For comparison, pAI [33], array pulse volume (APV) [8], 2DL from contour analysis [9], and $r_{\mathrm{Lc}}$ from L-cube polynomial [10] are listed in Table 3.

All the indexes are calculated for pulse datasets with normalized amplitude from 0 to 1 and analyzed using oneway analysis of variance (ANOVA). A $P$ value less than 0.05 is considered statistically significant (Table 4).

According to statistical analysis, APV is unable to entirely differentiate taut and slippery pulses $(2.30 \pm 0.33$ vs. $2.18 \pm 0.13 ; P=0.2809>0.05$ ) by losing the spatial characteristics and trend of 3DPI. 2DL and $r_{\mathrm{Lc}}$ which include spatial characteristics of 3DPI, respectively, have significant statistical differences in normal and taut pulses, but they are not designed for describing the pulse's spatial waves containing more than one peak like a slippery pulse.

Apparently, normal, taut, and slippery pulse images can be differentiated by $\overline{r_{1}}$ with spatial characteristics of 3DPI. On the side, $\Delta r_{1}$ which reflects the property of spatiotemporal changes is also a recommendable index that well differentiates slippery pulse images from normal and taut pulse images. 


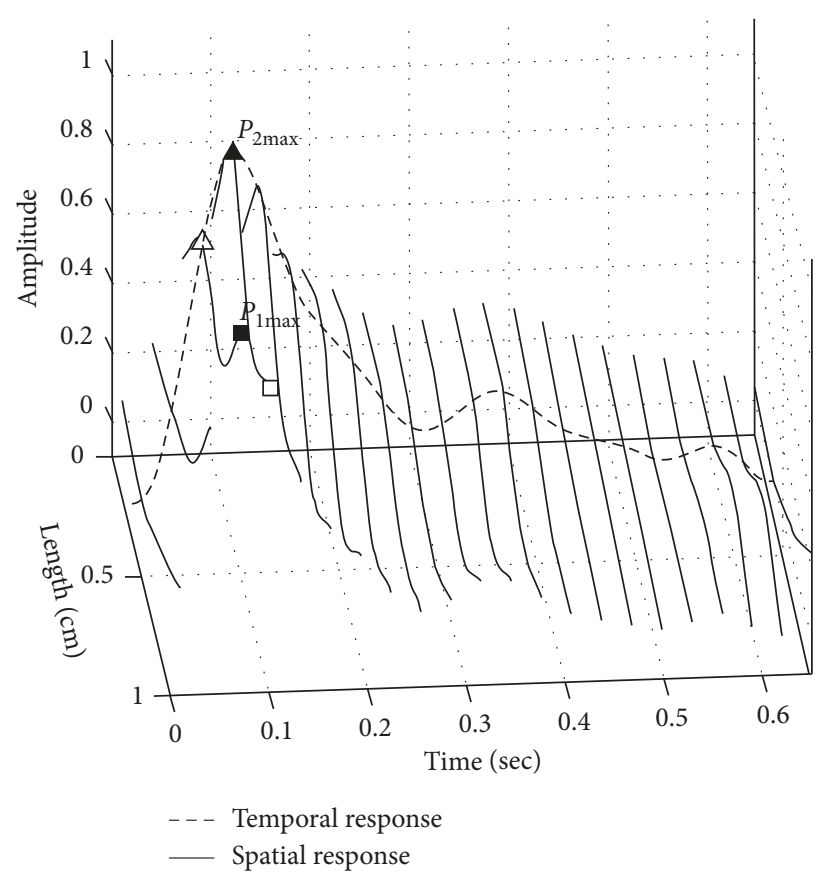

(a)

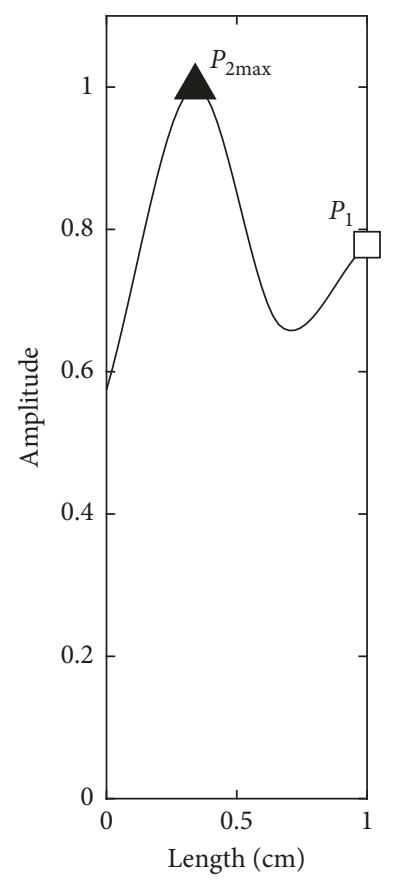

(c)

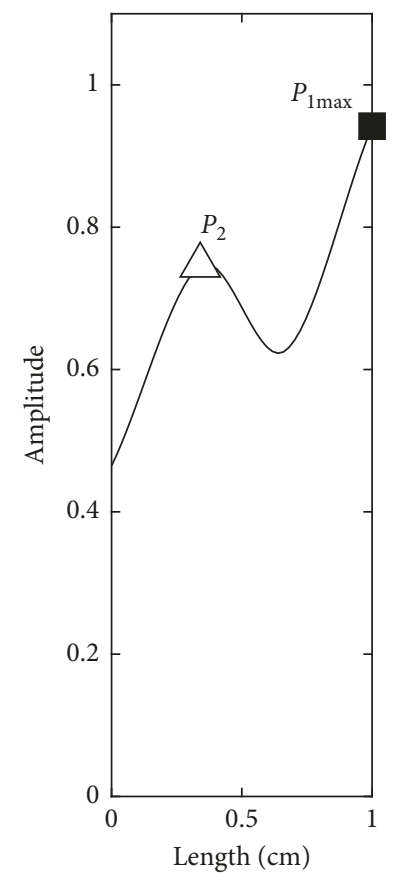

(b)

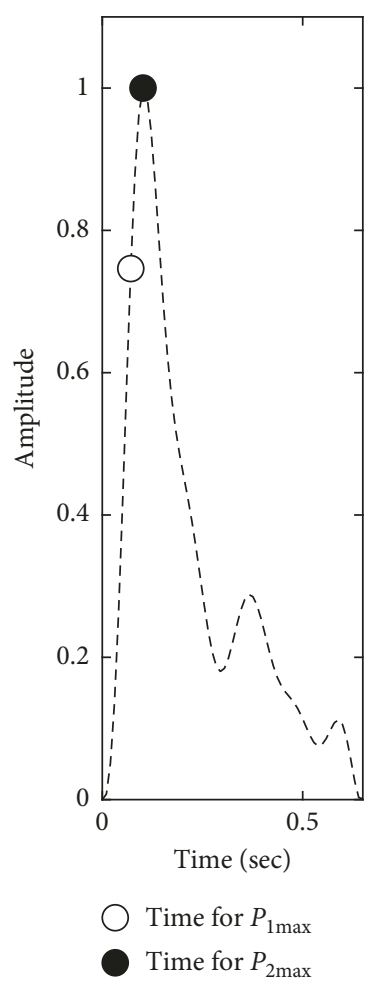

(d)

Figure 3: Temporal and spatial waves of a slippery d2DPI. (a) Temporal and spatial waves shown in a single pulse wave. (b) The first spatial peak maximum $\left(P_{1 \text { max }}\right.$, black rectangle). (c) The second spatial peak maximum $\left(P_{2 \max }\right.$, black triangle). (d) Time for $P_{1 \text { max }}$ (white circle) and $P_{2 \max }$ (black circle) in a temporal wave.

As an arterial stiffness index of a single temporal pulse wave [35], our results in Table 3 agree with the previous classification research that the pAI value of a normal pulse is higher than that of a slippery pulse while lower than that of a taut pulse [33]. It is worth noting that a taut pulse and a tight pulse in Chinese medicine are in similar shape and related to vessel tensing [32]. It is suggested that $\mathrm{CPT}$ on a healthy subject's hand and wrist $[9,36,37]$ can briefly increase 

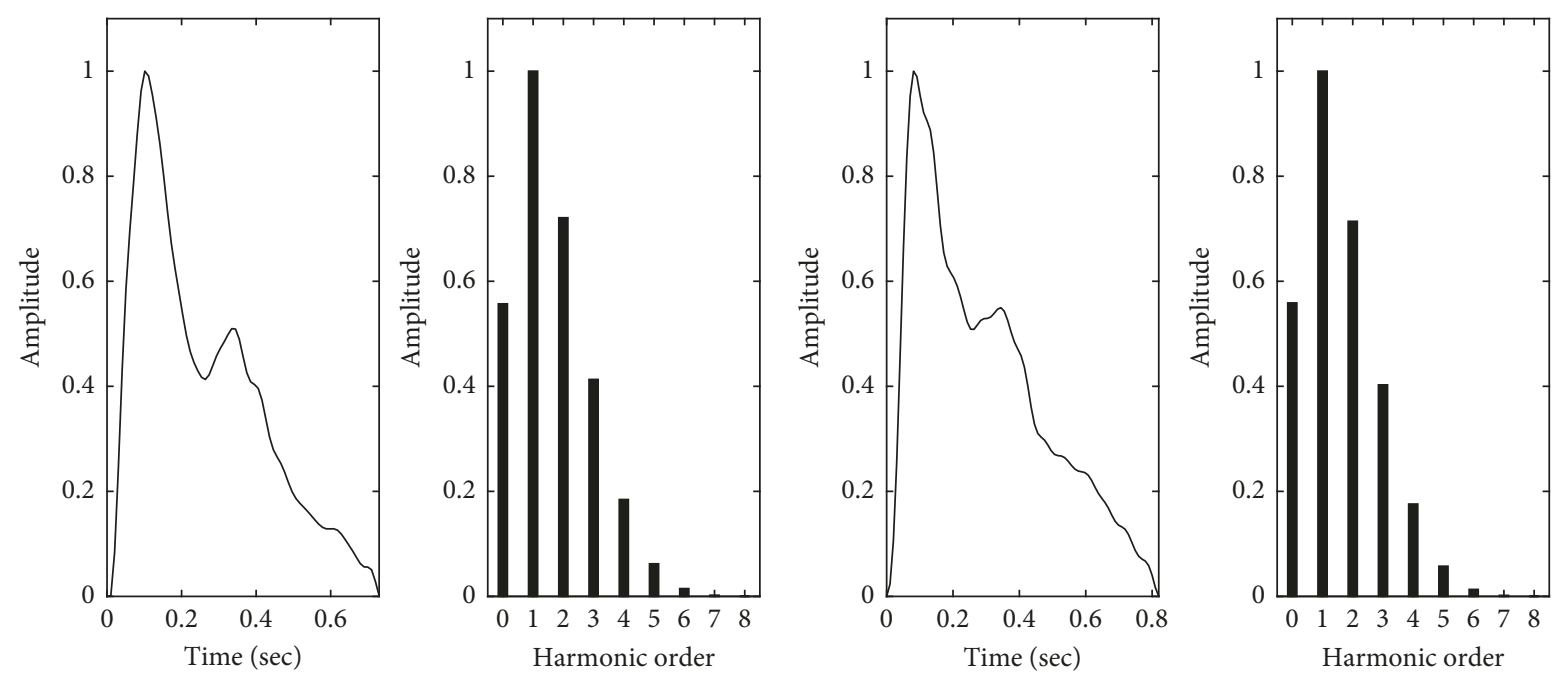

(a)
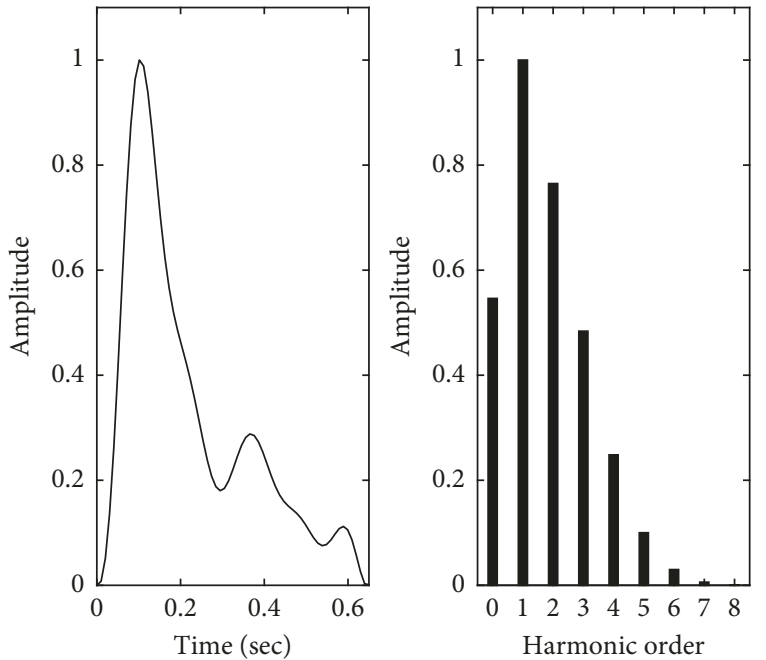

(c)

Figure 4: (a) Normal, (b) taut, and (c) slippery pulse's temporal wave (left) and their Fourier series (right).

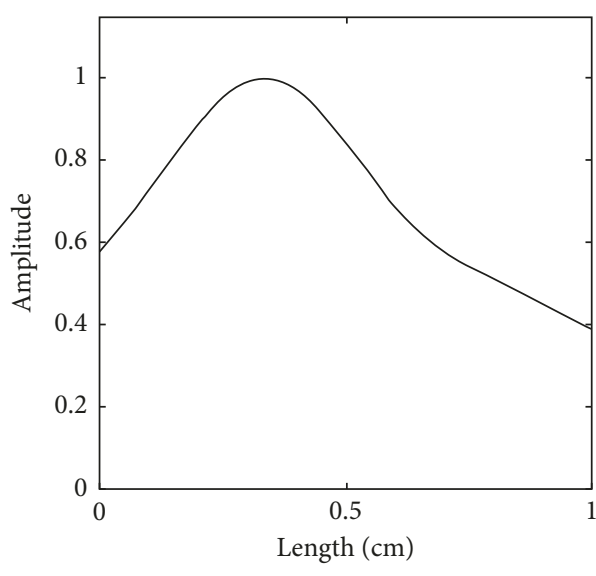

(a)

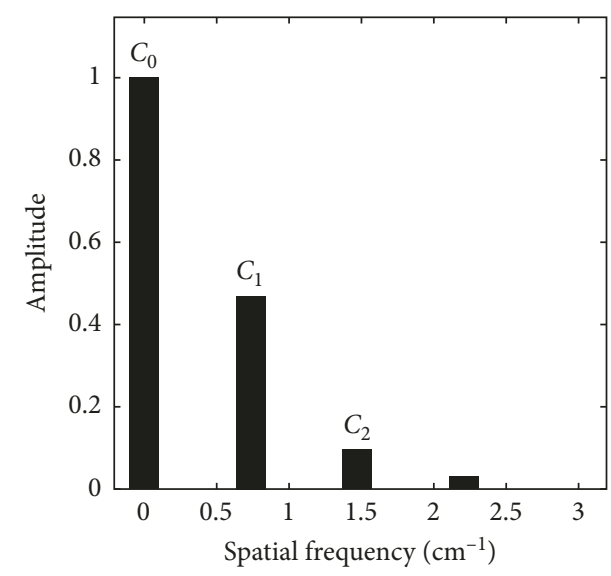

(b)

Figure 5: Normal pulse spatial wave (a) and its Fourier series (b). 


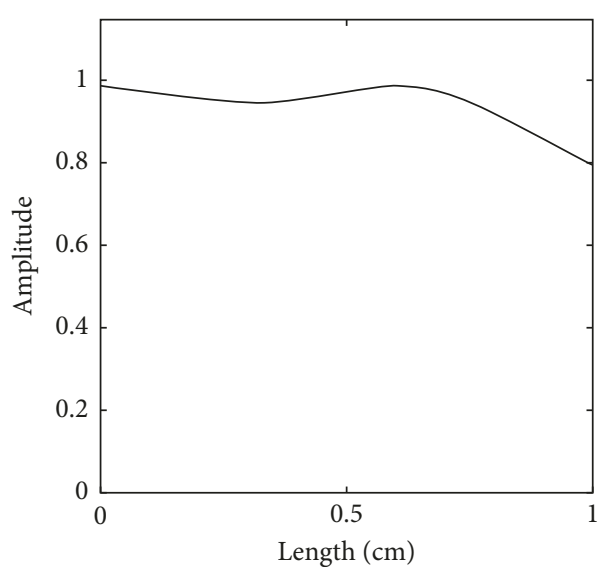

(a)

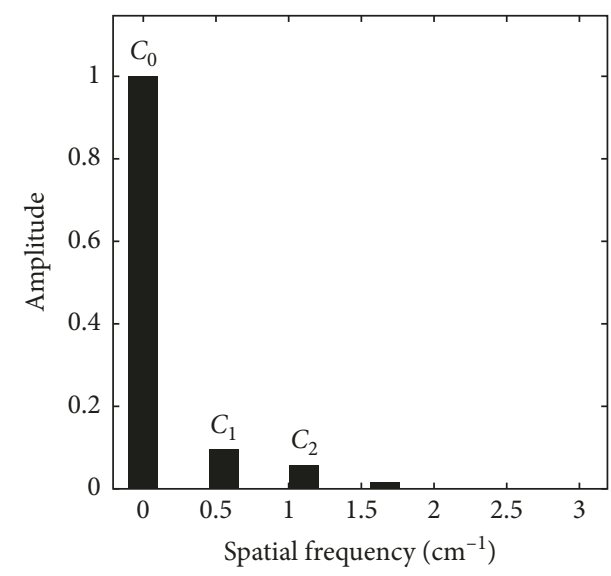

(b)

Figure 6: Taut pulse spatial wave (a) and its Fourier series (b).

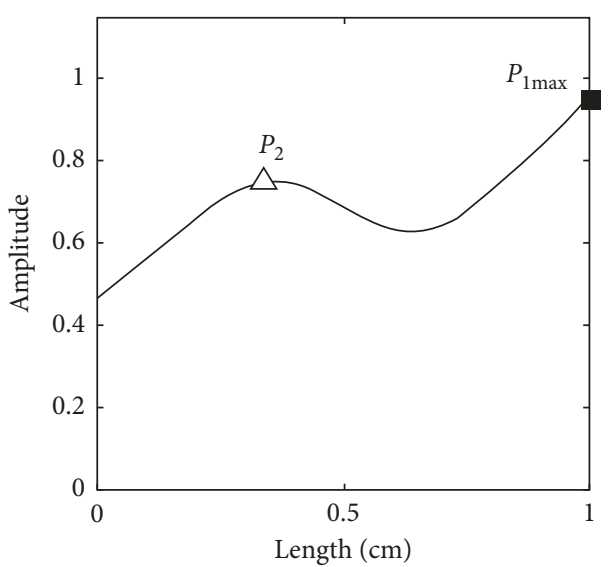

(a)

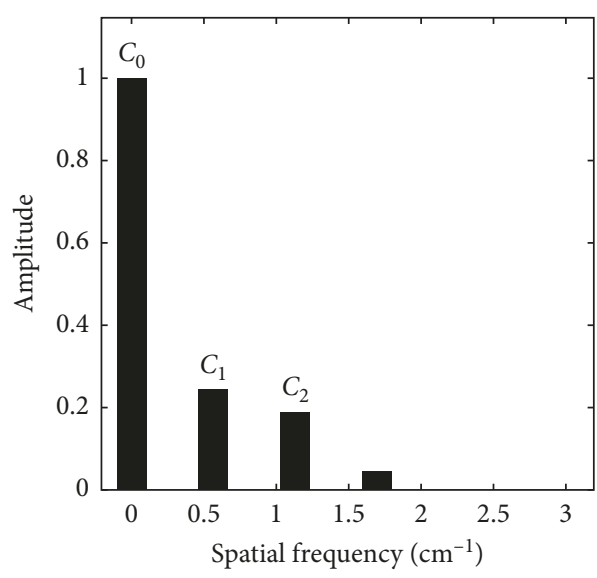

(b)

FIgURE 7: Slippery pulse spatial wave with the first peak (a) and its Fourier series (b).

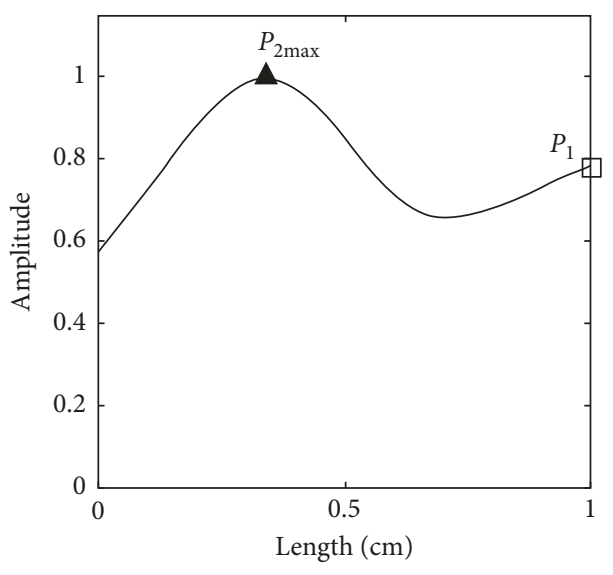

(a)

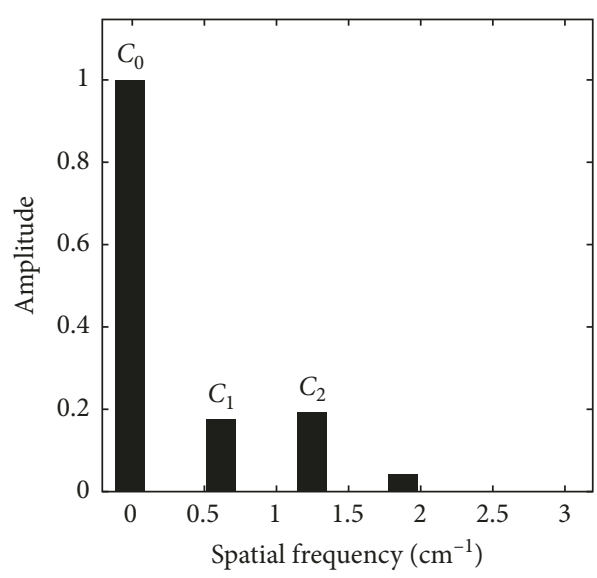

(b)

FIGURE 8: Slippery pulse spatial wave with the second peak (a) and its Fourier series (b). 


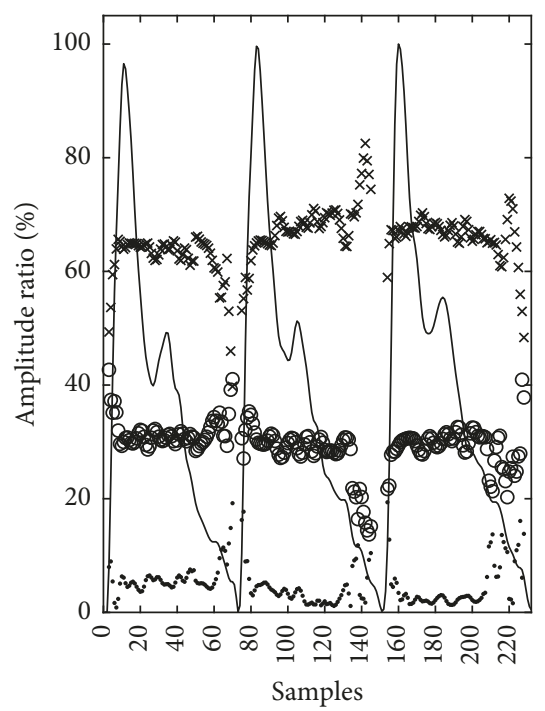

$\times \quad$ Sequence of $r_{0}$

- Sequence of $r_{1}$

- Sequence of $r_{2}$

_ Temporal response

(a)

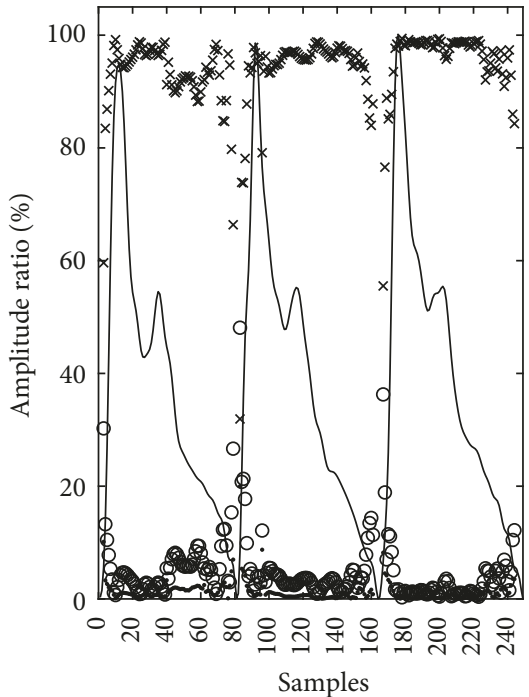

$\times$ Sequence of $r_{0}$

- Sequence of $r_{1}$

- Sequence of $r_{2}$

— Temporal response

(b)

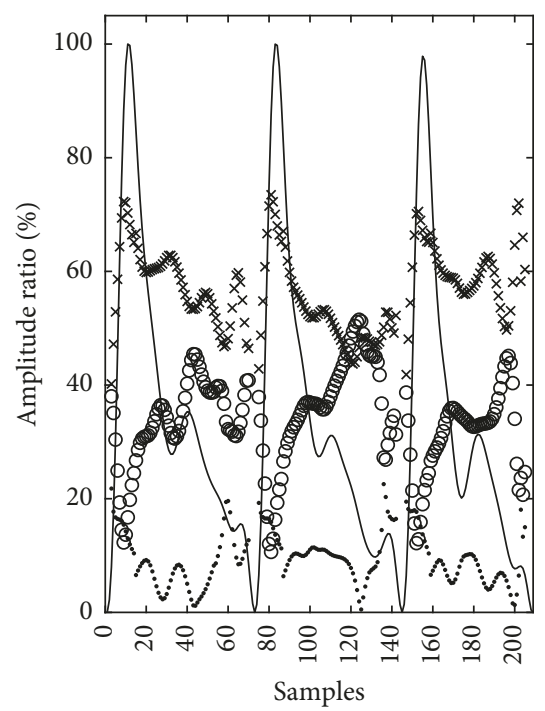

$\times$ Sequence of $r_{0}$

- Sequence of $r_{1}$

- Sequence of $r_{2}$

— Temporal response

(c)

FIGURE 9: Spatial harmonic ratio sequences of the Fourier series amplitudes: (a) normal pulse, (b) taut pulse, and (c) slippery pulse.

TABLE 2: Fourier series analysis of normal, taut, and slippery pulses.

\begin{tabular}{lccc}
\hline Pulse type & Temporal analysis & $\begin{array}{c}\text { Fourier series analysis of spatiotemporal pulse waves } \\
\text { Spatial analysis }\end{array}$ & Spatial harmonic ratio sequence \\
\hline $\begin{array}{l}\text { Normal pulse } \\
\text { Taut pulse }\end{array}$ & $\begin{array}{c}\text { Bndistinguishable and first harmonic share importance } \\
\text { Behaves as a rectangular pulse with dominant base } \\
\text { and little harmonics }\end{array}$ & $\begin{array}{c}\text { Three sequences are well distributed } \\
\text { Base is high, and others merged together }\end{array}$ \\
Slippery pulse & $\begin{array}{c}\text { The first harmonic can be higher or lower than the } \\
\text { second one }\end{array}$ & Three sequences intersect each other \\
\hline
\end{tabular}

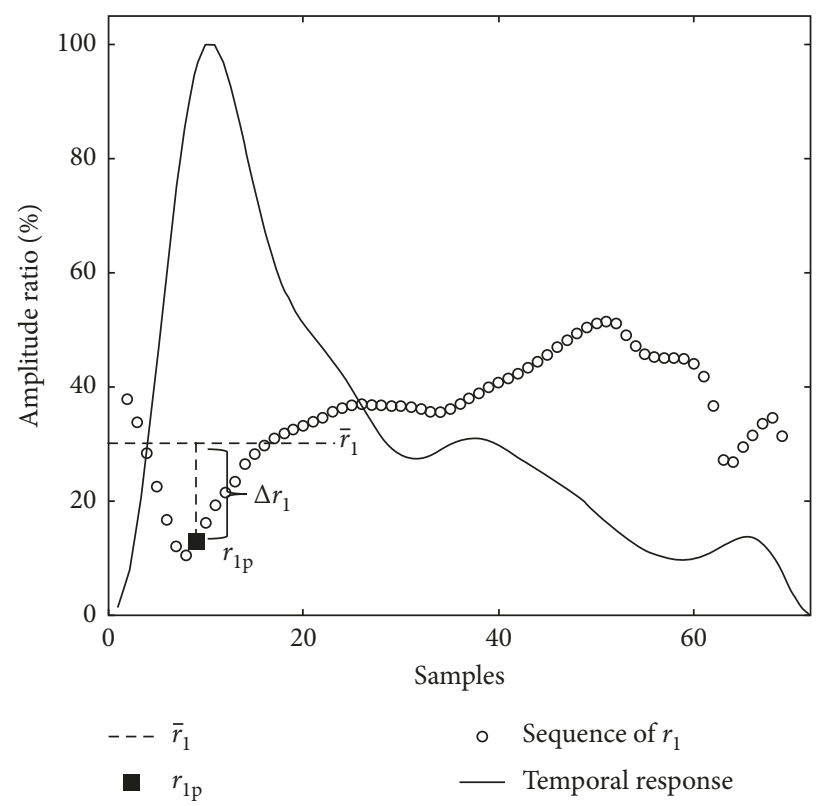

Figure 10: The relationship between the $\overline{r_{1}}, r_{1 \mathrm{p}}$, and $\Delta r_{1}$ in a slippery pulse. 
TABLe 3: Spatiotemporal pulse statistics.

\begin{tabular}{lccc}
\hline Methods and indexes & & Normalized value \\
& Normal pulse & Taut pulse & Slippery pulse \\
\hline pAI* [33] & $0.65 \pm 0.05$ & $0.75 \pm 0.07$ & $0.50 \pm 0.03$ \\
Array pulse volume [8] & $1.85 \pm 0.22$ & $2.30 \pm 0.33$ & $2.18 \pm 0.13$ \\
2DL from contour analysis [9] & $0.45 \pm 0.06$ & $0.75 \pm 0.11$ & - \\
$r_{\mathrm{Lc}}$ from L-cube polynomial [10] & $1.21 \pm 0.15$ & $2.14 \pm 0.44$ & - \\
{$\left[r_{1}, \Delta r_{1}\right]$ from the proposed method } & {$[0.39 \pm 0.04,0.00 \pm 0.02]$} & {$[0.25 \pm 0.05,0.03 \pm 0.04]$} & {$[0.32 \pm 0.03,0.16 \pm 0.03]$} \\
\hline
\end{tabular}

${ }^{*} \mathrm{pAI}$ is calculated from a single temporal pulse wave, not a spatial pulse wave.

Table 4: $P$ value from ANOVA test.

\begin{tabular}{lccc}
\hline Indexes & Normal-taut & $\begin{array}{c}P \text { value } \\
\text { Taut-slippery }\end{array}$ & Slippery-normal \\
\hline pAI & 0.0016 & 0.0000 & 0.0000 \\
APV & 0.0014 & 0.2809 & 0.0006 \\
$2 \mathrm{DL}$ & 0.0000 & - & - \\
$r_{\mathrm{LC}}$ & 0.0000 & - & - \\
$r_{1}$ & 0.0000 & 0.0009 & 0.0002 \\
$\Delta r_{1}$ & 0.0274 & 0.0000 & 0.0000 \\
\hline
\end{tabular}

arterial stiffness to induce a taut pulse, not a tight pulse, which fairly presents illness.

\section{Conclusions}

Fourier series is provided as a fast, robust, and real-time able method to analyze 3DPIs. It is difficult to differentiate these three types of pulses (normal, taut, and slippery) for the temporal Fourier series only because the definition of pulse feeling in Chinese medicine takes the spatial waveshape factor into account $[1,5,7]$. In comparison with temporal Fourier analysis in Table 2, the spatial Fourier analysis can distinguish among normal, taut, and slippery pulse images. For the Fourier series analysis of three spatiotemporal 3DPIs (normal, taut, and slippery), the proposed harmonic ratio sequence clearly shows apparently different behavior among three pulse images, and there are statistical differences between them by $\overline{r_{1}}$ and $\Delta r_{1}$ indexes.

In this study, taut pulses were induced by CPT, not collected from hypertensive patients. Normal and slippery pulse images were obtained from young people. In the near future, lots of 3DPI should be collected from patients with different ages and several kinds of hypertension to testify the statistical recognition rate of the proposed ratio sequence. Furthermore, various methods such as WT and HHT can be introduced to study the spatiotemporal characteristics of all types of 3DPI, not limited only with normal, taut, and slippery pulse images.

\section{Data Availability}

The data used to support the findings of the current study are available from the corresponding author on reasonable request.

\section{Conflicts of Interest}

The authors declare that there are no conflicts of interest regarding the publication of this paper.

\section{Acknowledgments}

This research was supported by National Cheng Kung University, Taiwan, under NCKU Top University Program, and Sun Yat-sen University, China, under Scientific Initiation Project no. 67000-18821109 for high-level experts.

\section{References}

[1] C.-S. Hu, Y.-F. Chung, C.-C. Yeh, and C.-H. Luo, “Temporal and spatial properties of arterial pulsation measurement using pressure sensor array," Evidence-Based Complementary and Alternative Medicine, vol. 2012, pp. 1-9, 2012.

[2] D. M. Wang, D. Zhang, and G. M. Lu, "A novel multichannel wrist pulse system with different sensor arrays," IEEE Transactions on Instrumentation and Measurement, vol. 64, no. 7, pp. 2020-2034, 2015.

[3] K. W. Kong, W. M. Lau, K. S. Wong et al., "A pulse-sensing robotic hand for tactile arterial palpation," in Proceedings of the 2016 IEEE International Conference on Cyber Technology in Automation, Control, and Intelligent Systems (Cyber), pp. 141-145, Chengdu, China, June 2016.

[4] C.-H. Luo, Y.-F. Chung, C.-C. Yeh et al., "Stringlike pulse quantification study by pulse wave in $3 \mathrm{D}$ pulse mapping," The Journal of Alternative and Complementary Medicine, vol. 18, no. 10, pp. 924-931, 2012.

[5] Y.-F. Chung, C.-S. Hu, C.-C. Yeh, and C.-H. Luo, "How to standardize the pulse-taking method of traditional Chinese medicine pulse diagnosis," Computers in Biology and Medicine, vol. 43, no. 4, pp. 342-349, 2013.

[6] Y.-W. Chu, C.-H. Luo, Y.-F. Chung, C.-S. Hu, and C.-C. Yeh, "Using an array sensor to determine differences in pulse diagnosis-Three positions and nine indicators," European Journal of Integrative Medicine, vol. 6, no. 5, pp. 516-523, 2014.

[7] C.-H. Luo, C.-J. Su, T.-Y. Huang, and C.-Y. Chung, "Noninvasive holistic health measurements using pulse diagnosis: I. Validation by three-dimensional pulse mapping," European Journal of Integrative Medicine, vol. 8, no. 6, pp. 921-925, 2016.

[8] J. Cui, L.-P. Tu, J.-F. Zhang, S.-L. Zhang, Z.-F. Zhang, and J.-T. Xu, "Analysis of pulse signals based on array pulse volume," Chinese Journal of Integrative Medicine, vol. 25, no. 2, pp. 103-107, 2019.

[9] C. J. Su, T. Y. Huang, and C. H. Luo, "Arterial pulse analysis of multiple dimension pulse mapping by local cold stimulation for arterial stiffness," IEEE Sensors Journal, vol. 16, no. 23, pp. 8288-8294, 2016.

[10] C.-H. Luo, J.-W. Ye, C.-Y. Lin, T.-L. Lee, L.-M. Tsai, and M.-D. Shieh, "L-cube polynomial for the recognition of normal and hypertensive string-like pulse mappings in 
Chinese medicine," Informatics in Medicine Unlocked, vol. 12, pp. 27-33, 2018.

[11] J. Y. A. Foo, "Use of independent component analysis to reduce motion artifact in pulse transit time measurement," IEEE Signal Processing Letters, vol. 15, pp. 124-126, 2008.

[12] H. F. Yang, Q. Zhou, and J. Xiao, "Relationship between vascular elasticity and human pulse waveform based on FFT analysis of pulse waveform with different age," in Proceedings of the 2009 3rd International Conference on Bioinformatics and Biomedical Engineering, vol. 1-11, pp. 254-257, Beijing, China, June 2009.

[13] B. Thakker and A. L. Vyas, "Frequency domain analysis of radial pulse in abnormal health conditions," in Proceedings of the 2010 IEEE EMBS Conference on Biomedical Engineering and Sciences (IECBES), pp. 227-231, Kuala Lumpur, Malaysia, December 2010.

[14] Y. Y. L. Wang, T. L. Hsu, M. Y. Jan et al., "Theory and applications of the harmonic analysis of arterial pressure pulse waves," Journal of Medical and Biological Engineering, vol. 30, no. 3, pp. 125-131, 2010.

[15] M. Saito, M. Matsukawa, T. Asada, and Y. Watanabe, "Noninvasive assessment of arterial stiffness by pulse wave analysis," IEEE Transactions on Ultrasonics, Ferroelectrics, and Frequency Control, vol. 59, no. 11, pp. 2411-2419, 2012.

[16] A. C. Tang, J. W. Chung, and T. K. Wong, "Validation of a novel traditional Chinese medicine pulse diagnostic model using an artificial neural network," Evidence-Based Complementary and Alternative Medicine, vol. 2012, Article ID 685094, 7 pages, 2012.

[17] D.-G. Jang, S.-H. Park, and M. Hahn, "Enhancing the pulse contour analysis-based arterial stiffness estimation using a novel photoplethysmographic parameter," IEEE Journal of Biomedical and Health Informatics, vol. 19, no. 1, pp. 256-262, 2015.

[18] S. Emrani, T. S. Saponas, D. Morris et al., "A novel framework for pulse pressure wave analysis using persistent homology," IEEE Signal Processing Letters, vol. 22, no. 11, 2015.

[19] C. Fischer, B. Domer, T. Wibmer, and T. Penzel, "An algorithm for real-time pulse waveform segmentation and artifact detection in photoplethysmograms," IEEE Journal of Biomedical and Health Informatics, vol. 21, no. 2, pp. 372-381, 2017.

[20] R. Guo, Y. Wang, H. Yan et al., "Analysis and recognition of traditional Chinese medicine pulse based on the hilberthuang transform and random forest in patients with coronary heart disease," Evidence-Based Complementary and Alternative Medicine, vol. 2015, Article ID 895749, 8 pages, 2015.

[21] X. J. Hu, L. Zhang, J. T. Xu et al., "Pulse wave cycle features analysis of different blood pressure grades in the elderly," Evidence-Based Complementary and Alternative Medicine, vol. 2018, Article ID 1976041, 12 pages, 2018.

[22] Y. N. Tsai, Y. C. Huang, S. J. Lin et al., "Different harmonic characteristics were found at each location on TCM radial pulse diagnosis by spectrum analysis," Evidence-Based Complementary and Alternative Medicine, vol. 2018, Article ID 9018271, 10 pages, 2018.

[23] M. A. Motin, C. K. Karmakar, and M. Palaniswami, "Selection of empirical mode decomposition techniques for extracting breathing rate from PPG," IEEE Signal Processing Letters, vol. 26, no. 4, pp. 592-596, 2019.

[24] Y. C. Huang, Y. H. Chang, S. M. Cheng et al., "Applying pulse spectrum analysis to facilitate the diagnosis of coronary artery disease," Evidence-Based Complementary and Alternative Medicine, vol. 2019, Article ID 2709486, 10 pages, 2019.

[25] D. A. McDonald, "The relation of pulsatile pressure to flow in arteries," The Journal of Physiology, vol. 127, no. 3, pp. 533-552, 1955.
[26] J. R. Womersley, "Method for the calculation of velocity, rate of flow and viscous drag in arteries when the pressure gradient is known," The Journal of Physiology, vol. 127, no. 3, pp. 553-563, 1955.

[27] E. O. Attinger, A. Anné, and D. A. McDonald, "Use of Fourier series for the analysis of biological systems," Biophysical Journal, vol. 6, no. 3, pp. 291-304, 1966.

[28] J. A. Staessen, R. Fagard, L. Thijs, and A. Amery, "Fourier analysis of blood pressure profiles," American Journal of Hypertension, vol. 6, no. 2, pp. 184S-187S, 1993.

[29] K. A. Reddy, B. George, and V. J. Kumar, "Use of fourier series analysis for motion artifact reduction and data compression of photoplethysmographic signals," Ieee Transactions on Instrumentation and Measurement, vol. 58, no. 5, pp. 1706-1711, 2009.

[30] C. W. Chang, J. M. Chen, and W. K. Wang, "Development of a standard protocol for the harmonic analysis of radial pulse wave and assessing its reliability in healthy humans," Ieee Journal of Translational Engineering in Health and MedicineJtehm, vol. 3, 2015.

[31] D. E. Dick, J. E. Kendrick, G. L. Matson, and V. C. Rideout, "Measurement of nonlinearity in the arterial system of the dog by a new method," Circulation Research, vol. 22, no. 2, pp. 101-111, 1968.

[32] Z. Fei, Contemporary Sphygmology in Traditional Chinese Medicine, People's Medical Publishing House, Beijing, China, 2003, in Chinese.

[33] J. Cui, Z.-F. Fei, Z.-F. Zhang et al., "Classification parameter range of pulse graphs of normal pulse, slippery pulse and taut pulse," Shanghai Journal of Traditional Chinese Medicine, vol. 12, p. 4, 2016.

[34] X. Li, Z. Y. Fu, P. Qian et al., "Computerized wrist pulse signal diagnosis using gradient boosting decision tree," in Proceedings 2018 IEEE International Conference on Bioinformatics and Biomedicine (BIBM), pp. 1941-1947, Madrid, Spain, December 2018.

[35] L. J. Qiao, Z. Qi, L. P. Tu et al., "The association of radial artery pulse wave variables with the pulse wave velocity and echocardiographic parameters in hypertension," EvidenceBased Complementary and Alternative Medicine, vol. 2018, Article ID 5291759, 11 pages, 2018.

[36] D. L. Wood, S. G. Sheps, L. R. Elveback, and A. Schirger, "Cold pressor test as a predictor of hypertension," Hypertension, vol. 6, no. 3, pp. 301-306, 1984.

[37] M. A. Sanchez-Gonzalez, R. W. May, P. C. Brown, A. P. Koutnik, and F. D. Fincham, "Depressive symptoms contribute to increased wave reflection during cold pressor test in young adult men," American Journal of Hypertension, vol. 26, no. 6, pp. 778-783, 2013.

[38] C.-Y. Chung, Y.-W. Cheng, and C.-H. Luo, "Neural network study for standardizing pulse-taking depth by the width of artery," Computers in Biology and Medicine, vol. 57, pp. 26-31, 2015.

[39] L. Xu, D. Zhang, K. Wang, N. Li, and X. Wang, "Baseline wander correction in pulse waveforms using wavelet-based cascaded adaptive filter," Computers in Biology and Medicine, vol. 37, no. 5, pp. 716-731, 2007.

[40] S. McKinley and M. Levine, "Cubic spline interpolation," College of the Redwoods, vol. 45, no. 1, pp. 1049-1060, 1998.

[41] D. A. McDonald, Blood Flow in Arteries, Edward Arnold, London, UK, 2nd edition, 1974.

[42] C. Vlachopoulos, M. O'Rourke, and W. W. Nichols, McDonald's Blood Flow in Arteries: Theoretical, Experimental and Clinical Principles, CRC Press, Boca Raton, FL, USA, 2011. 


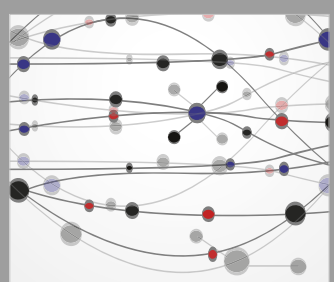

The Scientific World Journal
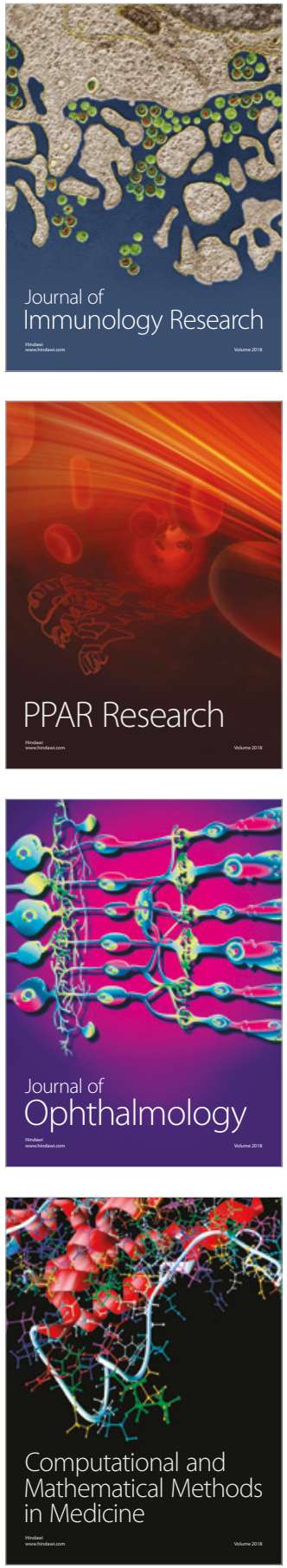

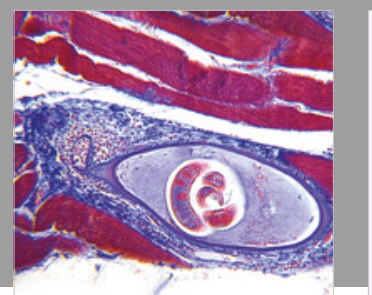

Gastroenterology Research and Practice

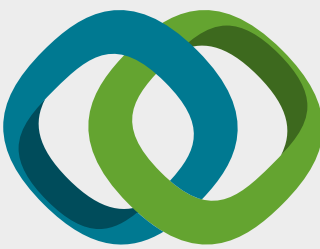

\section{Hindawi}

Submit your manuscripts at

www.hindawi.com
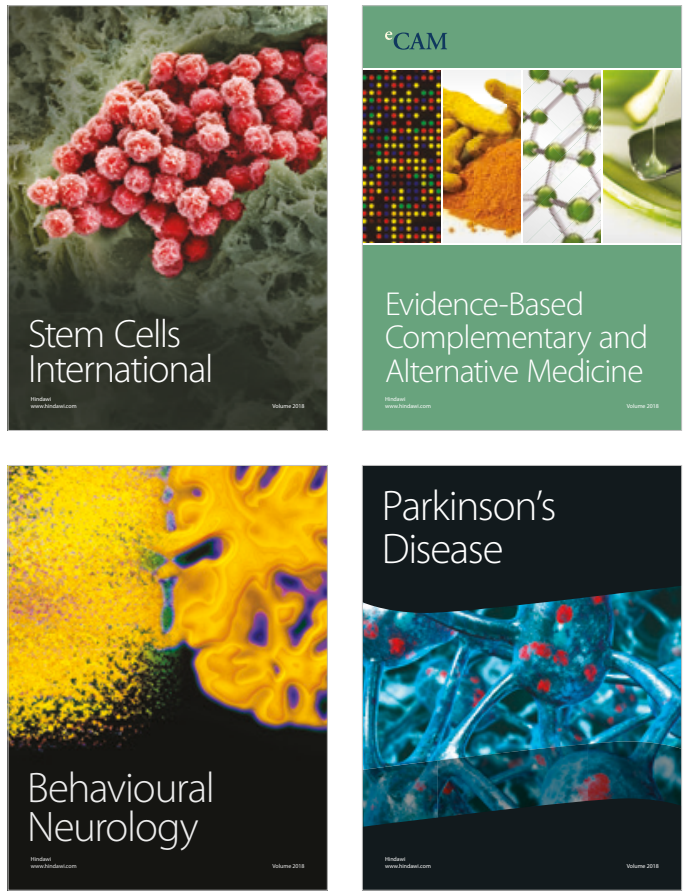

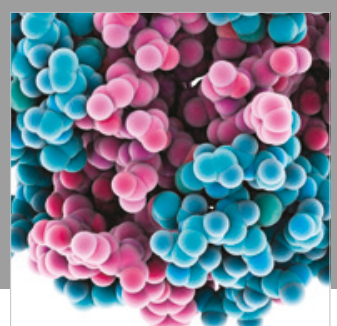

ournal of

Diabetes Research

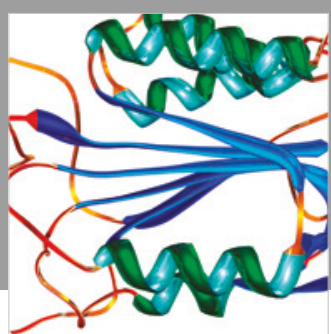

Disease Markers
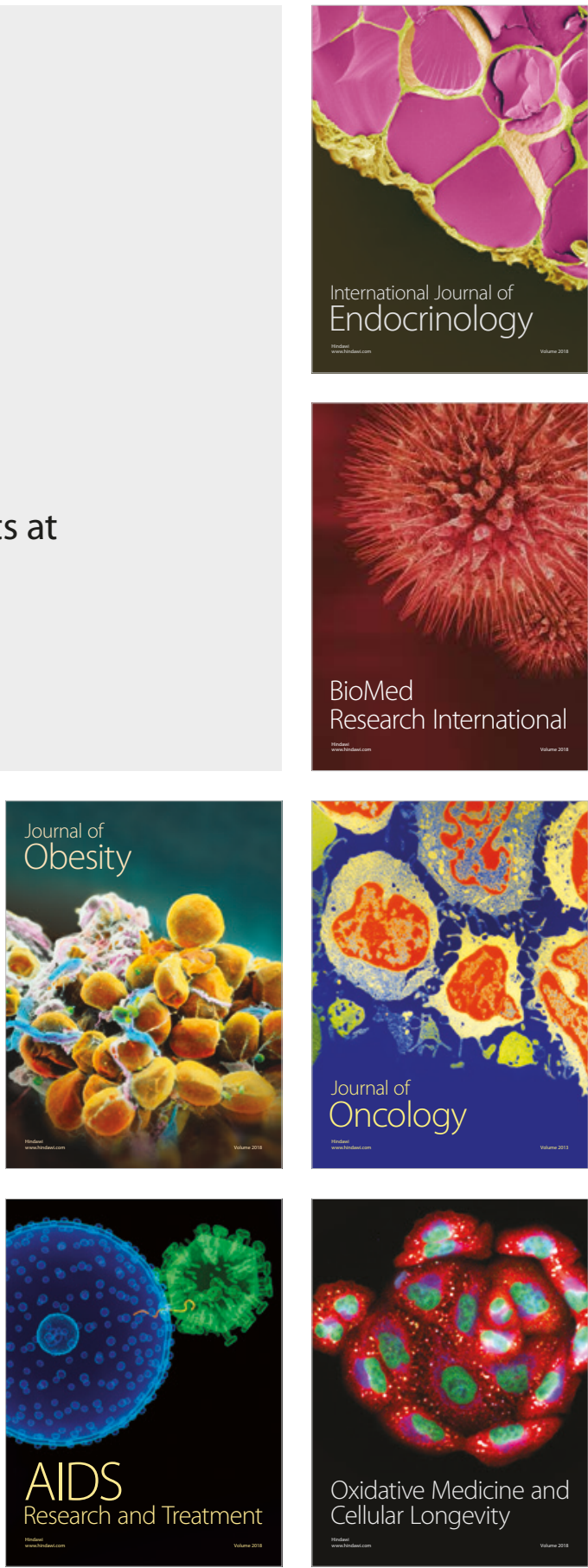